\title{
PROCESSAMENTO DO MELÃO POR MÉTODOS COMBINADOS: AVALIAÇÃO DAS CARACTERÍSTICAS MICROBIOLÓGICAS E FÍSICO- QUÍMICAS
}

\author{
MEN DE SÁ MOREIRA DE SOUZA FILHO * \\ TEREZINHA FEITOSA * \\ MANOEL ALVES SOUZA NETO ** \\ JOICE MELO WATERLOO *** \\ MARCELO MÜLLER ***
}

\begin{abstract}
Utilizou-se uma seqüência de métodos combinados em amostras de melão, a fim de determinar o tempo de armazenamento deste produto. $\mathrm{O}$ fruto foi submetido a branqueamento, seguido de osmose em diferentes concentrações de $\mathrm{SO}_{2}$, ácido sórbico, cloreto de cálcio e ácido cítrico até $\mathrm{pH}$ 3,0. As amostras foram embaladas em sacos de polietileno e tratadas termicamente em banho-maria a $100{ }^{\circ} \mathrm{C} / 1 \mathrm{~min}$. Durante o período de armazenamento foram avaliadas as características microbiológicas e físico-químicas do produto segundo recomendações da International Commission on Microbiological Specifications for Foods e da Association of Official Analytical Chemist. Os resultados obtidos indicaram que a tecnologia empregada para este tipo de produto assegura seu armazenamento pelo período de 120 dias. No entanto o elevado índice de perda de vitamina C evidencia necessidade de otimização do processo.
\end{abstract}

\section{INTRODUÇÃO}

O mercado mundial de produtos agrícolas envolve exportações num montante de US\$ 440 bilhões, dos quais o Brasil vende apenas US\$ 15 bilhões (18).

As estatísticas da Food and Agriculture Organization (FAO) mostram que o Brasil, mesmo posicionado como o maior produtor do mundo de frutas in natura, não está entre os vinte maiores exportadores de frutas frescas, destinando apenas $1 \%$ de sua produção para o exterior (16).

* Pesquisadores da Empresa Brasileira de Pesquisa Agropecuária (EMBRAPA), Centro Nacional de Pesquisa de Agroindústria Tropical (CNPAT), Fortaleza, CE.

** Assistente de pesquisa da EMBRAPA/CNPAT, Fortaleza, CE.

*** Bolsista da EMBRAPA/CNPAT, Fortaleza, CE. 
Embora apresente características promissoras, a fruticultura brasileira depara-se com vários pontos de estrangulamento. Uma das dificuldades que afetam o setor está relacionada ao enorme volume de perdas. Parte destas ocorre no próprio pomar, antes e durante a colheita, geralmente feita de maneira bastante rudimentar. No período de pós-colheita, o problema é maior, pois o produto é bastante perecível e suscetível a doenças. Agravam ainda mais a situação, o manuseio, o acondicionamento e o transporte normalmente feitos de maneira inadequada, aumentando o volume de perdas e diminuindo a qualidade das frutas que chegam ao consumidor (8).

Nos últimos anos foi desenvolvida tecnologia de preservação de alimentos, baseada no "efeito de obstáculos", que vem sendo bastante usada na preservação de frutas e hortaliças. Esta tecnologia consiste em aplicar, de forma branda, vários fatores de "stress" sobre o produto, com a finalidade de controlar o desenvolvimento microbiano e assim aumentar sua vida de prateleira. Além disso, possibilita a preservação das características do produto fresco, minimizando os custos de produção $(3,9,12,13)$.

Este trabalho foi realizado com o objetivo de estudar a vida útil do melão, processado minimamente, durante 120 dias.

\section{MATERIAL E MÉTODOS}

Foram utilizadas amostras de melão procedentes do mercado varejista da cidade de Fortaleza.

O estudo foi realizado em duas etapas. Na primeira fase testou-se o tempo ideal de branqueamento em um lote de melão. Após a definição deste tempo, outro lote de amostras foi processado conforme descrito no Fluxograma 1.

As amostras foram previamente lavadas com água clorada a 500 ppm, por imersão e selecionadas manualmente para posterior corte em pedaços uniformes. Em seguida efetuou-se branqueamento sob vapor a $100{ }^{\circ} \mathrm{C} / 2$ minutos.

Para a estabilização da atividade de água (Aw) foi utilizado xarope de sacarose a $25^{\circ} \mathrm{Brix}$, adicionado de $300 \mathrm{ppm}$ de $\mathrm{SO}_{2}, 1000 \mathrm{ppm}$ de ácido sórbico, $200 \mathrm{ppm}$ de cloreto de cálcio e ácido cítrico até pH 3, numa proporção fruto : xarope de 1:2, durante 5 dias. As amostras foram posteriormente embaladas em sacos de polietileno, termo-seladas, tratadas termicamente em banho-maria $\left(100^{\circ} \mathrm{C} / 1 \mathrm{~min}\right.$.) e armazenadas em temperatura ambiente. 


\section{FLUXOGRAMA 1 - ETAPAS DO PROCESSAMENTO DO MELÃO POR MÉTODOS COMBINADOS}

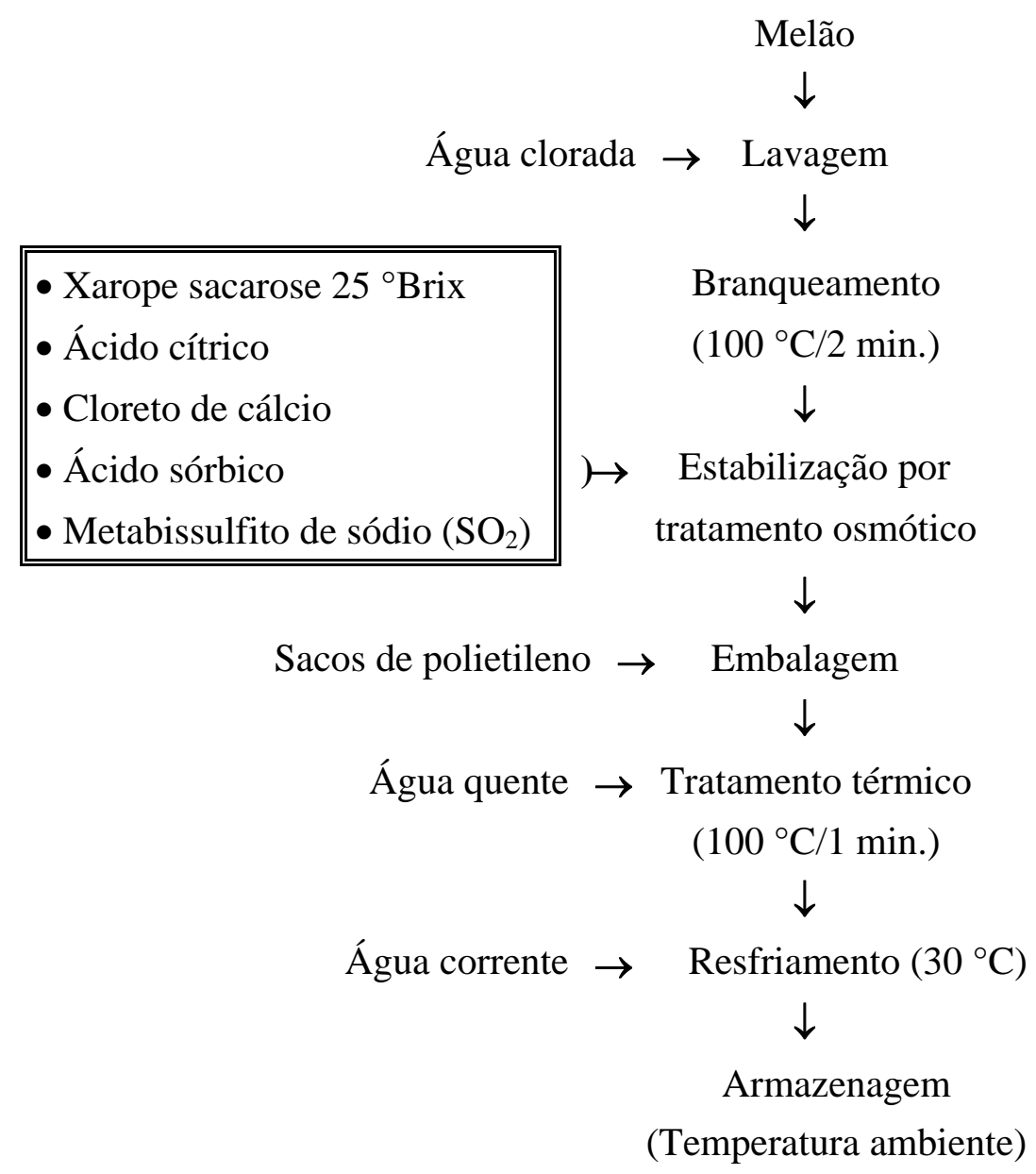

Para determinação da atividade da água (AW) do melão in natura e após tratamento osmótico, utilizou-se medidor Aw-wert Messer, modelo 5803 (Glufft mebund Regelltechnik $\mathrm{Gm}$ bH). A atividade de água do xarope selecionado para o tratamento osmótico das amostras foi estimada pela equação de Norrish, conforme CHIRIFE et al. (7), e a Aw do melão após o equilíbrio do sistema, pela equação de Ross, descritas abaixo:

\section{- Equação de NORRISH: $\quad A w_{(\text {soluto })}=X_{1}$. $\operatorname{EXP}\left[-K\left(X_{2}\right)^{2}\right]$}

Onde: $X_{1}$ e $X_{2}$ são as frações molares da água e do soluto $\mathrm{K}$ é a constante do soluto
- Equação de ROSS:
$A w_{(\text {(equilibrio) }}=A w_{\text {(fruto) }} \cdot A w_{\text {(sol. de soluto) }}$ 
As características físico-químicas de $\mathrm{pH}$ e sólidos solúveis ( ${ }^{\circ} \mathrm{Brix}$ ) foram avaliadas conforme as normas analíticas do INSTITUTO ADOLFO LUTZ (10). Determinou-se a umidade conforme AOAC (6), o teor de Vitamina C de acordo com PEARSON (17) e as análises microbiológicas seguiram as recomendações da International Commission on Microbiological Specifications for Foods (11) e da American Public Health Association (1).

\section{RESULTADOS E DISCUSSÃO}

$\mathrm{O} \mathrm{pH}$ das amostras variou de 6,42 na fruta in natura a 3,85 imediatamente depois do período de osmose, estabilizando-se em 3,62 após o armazenamento a temperatura ambiente, pelo período de 30 dias (Tabela 1). Esta diminuição do pH foi atribuída à adição de ácido cítrico, empregado como variante do processamento. O conteúdo de ácido ascórbico encontrado no fruto in natura foi de $31,47 \mathrm{mg} / 100 \mathrm{~g}$ (Tabela 2). A variação do teor desta vitamina nos frutos ocorreu em função de diversos fatores como práticas de cultivo, regime pluvial, exposição ao sol, tratamentos tecnológicos e outros $(1,15)$. Observou-se que durante as diversas fases do processamento houve perda de vitamina $C$, sendo que 0 tratamento osmótico foi o que proporcionou o mais alto nível de perda. ALZAMORA et al. (2) e VIAL et al. (19) também verificaram perdas de ácido ascórbico durante tratamentos osmóticos em diversos frutos.

\section{TABELA 1 - VARIAÇÃO DO pH DO MELÃO DURANTE 0 PROCESSAMENTO E ARMAZENAMENTO}

\begin{tabular}{c|c|c|c}
\hline Fruto “in natura” & Após osmose & $\begin{array}{c}\text { Após tratamento } \\
\text { térmico }\end{array}$ & $\begin{array}{c}\text { Após 30 dias de } \\
\text { armazenamento }\end{array}$ \\
\hline 6,42 & 3,85 & 3,79 & 3,62 \\
\hline
\end{tabular}

A cinética de degradação pode ser explicada pela combinação de fenômenos difusionais e degradação química. VIAL et al. (19) em trabalho sobre a desidratação osmótica de Kiwi, a baixa temperatura, constataram que os fenômenos difusionais constituíram a causa predominante na perda de ácido ascórbico.

Dentre os valores de umidade e atividade de água obtidos nota-se variação de $94,62 \%$ e 0,9600 , respectivamente no fruto in natura (Tabela 3), para $78,97 \%$ e 0,935 no fruto após tratamento térmico. Estes resultados assemelham-se aos obtidos por ALZAMORA et al. (2) e ARGAIZ et al. (5), os quais enquadram alimentos conservados por métodos combinados como sendo os que apresentam umidade entre $65 \%$ a $85 \%$ e Aw de 0,90 a 0,97 . 


\section{TABELA 2 - TEOR DE ÁCIDO ASCÓRBICO NO MELÃO IN NATURA, DURANTE O PROCESSAMENTO POR MÉTODOS COMBINADOS}

\begin{tabular}{l|c|c}
\hline \multicolumn{1}{c|}{ Amostra } & $\begin{array}{c}\text { Conteúdo de ácido } \\
\text { ascórbico }(\mathrm{mg} / 100 \mathrm{~g})\end{array}$ & $\begin{array}{c}\text { Perda de ácido ascórbico } \\
\mathbf{( \% )}\end{array}$ \\
\hline Fruto “in natura” & 31,47 & ZERO \\
\hline $\begin{array}{l}\text { Fruto após } \\
\text { branqueamento }\end{array}$ & 26,40 & 16,11 \\
\hline Após $1^{0}$ dia de osmose & 12,31 & 60,88 \\
\hline Após $5^{0}$ dia de osmose & 4,64 & 85,82 \\
\hline Após tratamento térmico & 3,50 & 88,87 \\
\hline
\end{tabular}

Em relação ao teor de sólidos solúveis (Tabela 3) observou-se acréscimo de $200 \%$ entre os valores iniciais obtidos para o fruto in natura $\left(7,0^{\circ} \mathrm{Brix}\right) \mathrm{e}$ ao final de 30 dias de armazenamento $\left(21,0^{\circ} \mathrm{Brix}\right)$.

TABELA 3 - SÓLIDOS SOLÚVEIS ('BRIX) NO MELÃO IN NATURA, DURANTE E APÓS O PROCESSAMENTO

\begin{tabular}{l|c}
\hline Fruto & Sólidos solúveis ( ${ }^{\circ}$ Brix $)$ \\
\hline in natura & 7,00 \\
Após 5 dias de osmose & 20,10 \\
Após tratamento & 21,00 \\
térmico/armazenamento & \\
\hline
\end{tabular}

De acordo com os resultados apresentados na Tabela 4 observa-se que, o branqueamento reduziu significativamente a contagem microbiana presente na matéria, e que o tempo de branqueamento a partir de 1,0 minuto foi suficiente para diminuir a contagem de bactérias mesófilas, bolores, leveduras e coliformes. Resultados semelhantes foram obtidos por ALZAMORA et al. (2) e LOPEZ MALO (14) em estudos com abacaxi e mamão. Demonstra-se assim que a estabilidade microbiológica (Tabela 5) pode ser obtida em altos níveis de atividade de água, desde que sejam usados, simultaneamente, vários fatores combinados de preservação, sem alterar as características naturais do fruto. Nesta pesquisa, além do 
branqueamento, foram utilizados $\mathrm{SO}_{2}$ e ácido sórbico, que previnem o escurecimento enzimático do produto e apresentam atividade antimicrobiana, bem como ácido cítrico para ajustar o pH do mesmo, tornando o processo mais efetivo.

\section{TABELA 4 - EFEITO DO TEMPO DE BRANQUEAMENTO A $100{ }^{\circ} \mathrm{C}$ COM VAPOR LIVRE SOBRE AS CARACTERÍSTICAS DO MELÃO IN NATURA}

\begin{tabular}{l|c|c|c|c}
\hline \multirow{2}{*}{ Determinações } & \multirow{2}{*}{$\begin{array}{c}\text { Fruto } \\
\text { “in natura” }\end{array}$} & \multicolumn{3}{|c}{ Branqueamento (minuto) } \\
\cline { 3 - 5 } & $7,1 \times 10^{5}$ & $3,0 \times 10^{4}$ & $<10$ & $<10$ \\
\hline Contagem padrão (UFC/g) & $7,1 \times 0$ & 2,00 \\
\hline Bolores e leveduras (UFC/g) & $4,0 \times 10^{2}$ & $<15$ & $<10$ & $<10$ \\
\hline Coliformes totais (NMP/g) & $<3$ & $<3$ & $<3$ & $<3$ \\
\hline Coliformes fecais (NMP/g) & $<3$ & $<3$ & $<3$ & $<3$ \\
\hline
\end{tabular}

TABELA 5- CARACTERÍSTICAS MICROBIOLÓGICAS DO MELÃO CONSERVADO POR MÉTODOS COMBINADOS E ARMAZENADOS EM TEMPERATURA AMBIENTE

\begin{tabular}{l|c|c|c|c|c|c}
\hline \multirow{2}{*}{ Determinações } & Fruto & \multicolumn{5}{|c}{ Armazenamento (dias) } \\
\cline { 3 - 7 } & "in natura” & 0 & 30 & 60 & 90 & 120 \\
\hline Contagem padrão (UFC/g) & $1,6 \times 10^{5}$ & $3,0 \times 10^{4}$ & $<10$ & $<10$ & $<10$ & $<10$ \\
Bolores e leveduras (UFC/g) & $4,5 \times 10^{2}$ & 15 & $<10$ & $<10$ & $<10$ & $<10$ \\
Coliformes totais (NMP/g) & $1,1 \times 10^{3}$ & $<3$ & $<3$ & $<3$ & $<3$ & $<3$ \\
Coliformes fecais (NMP/g) & $<3$ & $<3$ & $<3$ & $<3$ & $<3$ & $<3$ \\
\hline
\end{tabular}

\section{CONCLUSÃO}

A análise do produto final permite concluir que, a combinação de métodos utilizados conferiu estabilidade às amostras armazenadas até 120 dias, em temperatura ambiente. Entretanto constatou-se elevada perda no teor de Vitamina "C" do fruto, evidenciando que o processo deve ser otimizado, a fim de preservar melhor as características nutritivas do melão. 


\begin{abstract}
It is studied a sequence of combined methods to assess their influences on the shelf-life of melon minimally processed. After blanching followed by osmotic treatment in different concentrations of $\mathrm{SO}_{2}$, sorbic acid, calcium chloride and citric acid to $\mathrm{pH} 3.0$, samples were packed in poliethylene bags and heat-treated in water $\left(100{ }^{\circ} \mathrm{C}\right)$ for 1 minute. During storage, samples were analyzed concerning microbiological and physical-chemical characteristics according to the ICMSF and the AOAC recommendations. Althought the results revealed that the used technology allowed a 120-day storage period for melon, the high depletion of vitamin $\mathrm{C}$ levels denotes a need of optimization in the process.
\end{abstract}

\title{
REFERÊNCIAS BIBLIOGRÁFICAS
}

1 ALVES, R.E., CHITARRA, A.B., CHITARRA, M.J.F. Postharvest physiology of acerola (Malphighia ermaginata D.C.) fruits: maturation changes; respiration activity and refrigerated storage at ambient and modified atmosphere. Lavras : ESALQ/Dep. Ciência de Alimentos, 1993. 7 p.

2 ALZAMORA, S.M., GERSCHENSON, N., CERRITI, P., ROJAS, A.M. Shelt stable pineapple for long-term non-refrigerated storage. Lebensem. Wiss. U. Technol., v. 22, p. 233-236, 1989.

3 ALZAMORA, S.M., TAPIA, M.S., ARGAIZ, A., WELTI, J. Application of combined methods technology in minimally processed fruit. Food Res. Int., v. 26, p. 125-130, 1993.

4 AMERICAN PUBLIC HEALTH ASSOCIATION. Compendium of methods for the microbiological examination of foods. 2.ed. Washington, D.C., 1984.

5 ARGAIZ, A., LOPEZ-MALO, A., WELTI, J., ALZAMORA, S.M. Fruit preservation by combined method. In: SYMPOSIUM ON MINIMALLY PROCESSED FRUIT AND VEGETABLE, New York, 1991. Procedings... New York : American Chemical Society, 1991. p. 234.

ASSOCIATION OF OFFICIAL ANALYTICAL CHEMIST. Official methods of the Association Of Official Analitical Chemists. 20.ed. Washington, 1975.

7 CHIRIFE, J., FERRO-FONTAN, C., BENMERGUI, E.A. The prediction of water activity in conection with intermediate moisture foods. J. Food Technol., v. 15, p. 59-70,1980.

8 FAZIO, G. Produção e consumo de frutas "in natura". Rev. de Administração, São Paulo, v. 29, n. 1, p. 83-88, jan./mar. 1994. 
9 GOULO, G.W., JONES, M.V. Combination and synergistic effect. In: MECANISMS of action food preservation procedures. London : Elsevier, 1989. p. 402-421.

10 INSTITUTO ADOLFO LUTZ. Métodos químicos e físicos para análise de alimentos. 3. ed. São Paulo, 1985. v. 1.

11 INTERNATIONAL COMMISSION ON MICROBIOLOGICAL SPECIFICATIONS FOR FOODS. Soft drinks, fruit preserves: food commodities. London : Academic Press, 1980. v. 2.

12 LEISTNER, L. Hurdle technology applied to meat products of the shelf stable products and intermediate moisture foods type. In. SIMATOS, D., MULTON, J.I. Properties of water in foods. Dordrecht, Netherlands : Martinus Nijhoff, 1985. p. 309, .

13 LEISTNER, L., RODEL, W., KRISPIEN, K. Microbiology of meat and meat products in high and intermediate moisture range. In: WATER activity: influence on food quality. New York : Academic Press, 1981. $855 \mathrm{p}$.

14 MONSAlVES, G.A., BARBOSA, C.G.V., CAVARIELE, R. P. Mass transfer and textural changes during processing of apple by combined methods. J. Food Sci., v. 58, p.1118-1124, 1993.

15 PEARSON, D. The chemical analysis of food. 6.ed. New York: Chemical Publ., 1971.

16 ROSS, K. D. Estimation of activity water in intermediate moisture foods. Food Technol., v. 29, n. 3, p. 26-39, 1975.

17 VIAL, C., GUILBERT, S., CVQ, J.L. Osmotic dehydration of kiwi fruits influence of process variables on the color and ascorbic acid contet. Science des Aliments, v. 11, p. 63-84, 1991. 